\title{
25 Research Soure \\ The effect of Alpha-lipoic acid in glyphosate treated granulosa cells on the human folliculogenesis genes
}

Sakineh Kaboli Kafshgiri

Birjand University of Medical Sciences

Armin Attaranzadeh

Mashhad University of Medical Sciences

Fahimeh Ghasemi

Birjand University of Medical Sciences

Ebrahim miri-moghaddam ( $\square$ Moghaddam4@yahoo.com )

Birjand University of Medical Sciences

\section{Research Article}

Keywords: Alpha-lipoic acid, Glyphoste, Human granulosa cell, FOXO1, NOX4, STAR, Vnn1

Posted Date: May 11th, 2021

DOl: https://doi.org/10.21203/rs.3.rs-436334/v1

License: (a) (i) This work is licensed under a Creative Commons Attribution 4.0 International License. Read Full License 


\section{Abstract}

Pesticides have a wide range of infertility in female reproductive. GLP is the most common herbicide used worldwide. The present study tended to evaluate the effects of alpha-lipoic acid (ALA) on expression of folliculogenesis genes in human granulosa cells (GCs) treated with glyphosate. In this study, GC samples were taken from infertile male patients who had tubal factors and received ICSI or IVF treatment for the first time. The medium used to culture cells was DMEM-F12 containing FBS 10\%, penicillin 1\%, and streptomycin $1 \%$. The cells were treated with glyphosate (GLP) $(250 \mu \mathrm{g} / \mathrm{ml})$, ALA $(50 \mu \mathrm{g} / \mathrm{ml})$, and their mixture (250 GLP + 50 ALA) for $24 \mathrm{hr}$. The Fox01, NOX4, Vnn1, and STAR gene expression in GCs was determined by real-time PCR. Exposure to GLP decreased gene expression Vnn1 and STAR in the GCs, while in comparison to control group, FOXO1 and NOX4 expression insignificantly increased. Further, ALA treatment decreased FOX01, NOX4, in the GCs, and increased VNN1 and STAR gene expression. Transcriptional expression of Fox01, NOX4 and STAR in GCs also decreased in their combinations (GLY + ALA). At the same time, the level of Vnn1 mRNA in GCs was increased. The findings showed that exposure to GLP alters the morphological structure of GCs and the expression of follicogenic genes, leading to dysfunction of the reproductive system. Moreover, the results showed that cell characteristics were preserved efficiently in human GCs exposed to ALA as an antioxidant.

\section{Introduction}

Infertility is still a widespread problem, affecting more than $10 \%$ of female population worldwide, as estimated (WHO 2017). In addition, one-third of infertile couples have no specific cause reported (Milesi et al., 2018). Recent studies suggest that certain lifestyle and increased levels of exposure to endocrinedisrupting chemicals (EDCs) may cause problems in reproductive system (Den Hond et al., 2015; Ziv-Gal et al., 2016). A close relationship was found by epidemiological studies between environmental or occupational exposure to pesticides and unpleasant disorders in reproductive systems of men and women (Chevrier et al., 2013; Mostafalou et al., 2017). Some adverse effects on reproductive system may include increased risk of infertility, abortion, stillbirth, low birth weight, or congenital anomalies (Chiu et al., 2015; Garca et al., 2017; Rappazzo et al., 2016).

Insecticides are important part of agricultural system; however, they affect animals and humans (Gupta et al., 2006; Mukhtar et al., 2013). Pesticides may interfere with female hormonal function and lead to dysfunction and infertility; they negatively affect the reproductive system; these negative effects may include gametogenesis, problems in ovulation and menstruation, infertility, miscarriage, ovarian disorders, and anomalies (Mokhtar et al., 2013; Saleh et al., 2014). Alternations may occur in survival granulosa cells (GCs) due to exposure to pesticides (Sargazi et al., 2015). A large group of chemicals which are used in agriculture as insecticides and acaricides is formed by organophosphorus (OP) compounds. OPs mainly target acetylcholinesterase in cholinergic synapses and neuromuscular junctions. GLP is the most common herbicide used in the world. As glyphosate-based herbicides (GBHs) are used in large scale, scientists are increasingly concerned with their toxic effects on animals and plants. Because GLP or N(phosphonomethyl) glycine is able to function as a potent weed-killer and it is widely used as herbicide, it 
is considered as one of the most significant chemicals in modern agriculture (Duke et al., 2008; Benbrook 2016). Recent studies showed that GLP is associated with many diseases such as cancer, metabolic disorders, endocrine disruption, imbalanced intestinal flora, autism spectrum disorders, and infertility; in addition, it mediates DNA damages, alters gene expression, and causes mutations (WHO 2017; Zhang et al., 2019).

Alpha-lipoic acid (ALA) is a natural chemical and an anti-inflammatory and potent antioxidant agent which is synthesized from octanoic acid and cysteine in human and animal mitochondria. Many studies showed that radical effects of ROS are eliminated as ALA fights oxidative stress (Armagan et al., 2015). Therapeutically, ALA is effective on many diseases associated with oxidative stress, including ischemiaperfusion injury, diabetes, cataract formation, neurodegeneration, HIV activation, and radiation damage (Huang et al., 2007; Karapinar et al., 2017).

Some genes play a role in developing the reproductive system and are necessary to develop gonads, including the testes and ovaries. One of the genes contributing to growth of female reproductive system is the vnn 1 I gene, which is an important indicator of follicle integrity. Transcription factors also have a subfamily known as Forkhead $\mathrm{O}$ (FoxO), which is responsible for regulation of wide-ranging cellular processes, including DNA repair, apoptosis, cell cycle arrest, cell differentiation, stress response, cell proliferation, metabolism, longevity, and senescence (Barthel et al., 2005). The role of FoxO transcription factors has been highlighted in mammalian ovaries. NOX4, a gene from NOX family, plays a significant role in many cellular systems. NOX 4 has been shown to be expressed in human GCs (Kampfer et al., 2014). Studies show that the NOX4 gene in GCs is a ROS production source, which may be related to female fertility factors. STRA (Steroidogenic Acute Regulatory Protein) gene is one of the steroidogenic that are regulated and expressed in GCs. The essential steroid enzyme in GCs is the STAR gene, which contribute to transfer of cholesterol from the outer mitochondrial membrane to the inner membrane and its conversion to pregnenolone.

A study on the effect of organochlorine pesticides on oocyte maturation of starfish and mouse found that females were more likely to get toxicated than males (Sharma et al., 2015). Little is known about the effect of these pesticides on human oocytes, granulosa, maturation, and fertilization. This study tends to evaluate the effects of ALA on expression of folliculogenesis genes, Foxo1, Vnn1, STAR, NOX4, in glyphosate-treated human GCs.

\section{Materials And Methods}

In this experimental-laboratory study, after obtaining informed written consent, luteal GCs (from the follicular fluid of 46 females) were isolated at the age of fewer than 40 years. Inclusion criteria included partners with total sperm number $<1 \times 106 / \mathrm{ml}$, motility rate of $<5 \%$, and abnormality index $>95 \%$, as specified by the World Health Organization (WHO 2010), as well as partners with tubular factor infertility. Exclusion criteria included patients with human immunodeficiency virus, hepatitis $C$ and $B$ virus, polycystic ovarian syndrome and cytomegalovirus infectivity. Healthy women with a history of in-vitro 
fertilization (IVF) or intracytoplasmic sperm injection (ICSI) were used to take cumulus oocyte-complexes (COCs). GCs collected as heaps in a $25 \mathrm{~cm}^{2}$ culture flask containing.

\section{Collection of human Granulosa Cells and cell culture}

GCs were taken from patients who had tubal or male infertility factors and underwent IVF or ICSI for the first time. Cumulus oocyte-complexes (COCs) were taken from ovary by an expert gynecologist using a transvaginal ultrasound-guided suction system. A fine and sterile pipet was used to isolate GCs and COCs in a pre-heated medium containing hyaluronidase (Cat No: $\mathrm{H} 1136$, Sigma) at $37^{\circ} \mathrm{C}$. GCs were mixed with an enzymatic solution containing hyaluronidase for $3 \mathrm{~h}$ and rinsed three times with phosphate-buffered saline. The cells were cultured in DMEM-F12 medium containing FBS $10 \%$ and penicillin $1 \%$ and streptomycin $1 \%$; finally, they were incubated at $37^{\circ} \mathrm{C}$ with $5 \% \mathrm{CO}_{2}$ for five days. The effects of GLP and ALA on GCs were determined by treatment of different concentrations of GLP (Bayer, Germany) $(250 \mu \mathrm{g} / \mathrm{ml})$ and ALA (Sigma, Germany) $(50 \mu \mathrm{g} / \mathrm{ml})$ and their combinations (250 GLP+ $50 \mathrm{ALA})$ for $24 \mathrm{hr}$.

\section{Cytomorphological study}

Morphological changes in GCs after treatment with GLP and ALA concentrations and simultaneous concentrations were evaluated by inverse microscopy.

\section{Evaluation of gene expression using real-time PCR}

Real-time PCR was used to evaluate FoxO1, NOX4, STAR, and Vnn1 mRNA expressions. As specified by the manufacturer, a high pure RNA isolation kit (Denazist, Iran) was used to extract the whole RNA from untreated and treated GCs; then, GCs were stored at $-80^{\circ} \mathrm{C}$. Then, mRNA was evaluated in terms of quality and quantity. A reverted first-strand cDNA synthesis kit (Thermo Scientific, USA) was used to synthesize CDNA, as specified by the manufacturer. Finally, Real-time PCR was run on $20 \mu$ mixture containing $10 \mu l$ SYBR Green master mix (Pars Tous, Iran), $1 \mu \mathrm{l} \mathrm{CDNA,} 8 \mu \mathrm{l}$ nuclease-free water and $1 \mu \mathrm{l}$ primer, as shown in Table 1, using a real-time PCR detection system (Bio-Rad CFX96). Expression levels were normalized using housekeeping gene GAPDH. Table 1 lists sequences of the primers used, (Table 1).

\section{Statistical analyses}

One-way ANOVA and Tukey test were used to analyze the data reported as mean \pm standard deviation ( $P$ $<0.05)$.

\section{Results}

\section{In-vitro studies}

Compared to the control group, GCs of the experimental group exhibited cell shrinkage, reduced cell volume, and plasma membrane distortion in exposed GLP and GLP + ALA group (each GLP concentration combined with each ALA concentration), while these were not observed in exposed ALA group. These 
characteristics of the cytoplasm and the shrinkage and deformation of the cells may indicate apoptosis in the GCs (Fig. 1).

\section{Effect of glyphosate, alpha-lipoic acid and their combination on Fox01, NOX4, Vnn1, and STAR expression in granulosa cells}

Real-time PCR was used to evaluate gene expression in GCs treated with GLP, ALA, and GLP + ALA. As data showed, increase in GLP concentrations elevated the transcription of FOXO1 and NOX4; compared to the control group, however, this increase was not significant. In comparison to the control group, it also decreased VNN1 and STAR expression in granulose cells. STAR gene expression significantly decreased $(P<0.01)$ compared to the control group. FOX01 and NOX4 expressions insignificantly decreased in the ALA group. At the same time, vnn1 and STAR expression increased. In comparison to the control group, Vnn1 expression significantly increased $(P<0.05)$; thus, FOXO1 and NOX4 expression decreased insignificantly in the simultaneous or synergistic treatment group or GLP + ALA group. Moreover, vnn1 expression increased insignificantly in the synergistic or GLP + ALA group compared to the control group, while STAR expression decreased significantly (Fig. 2).

\section{Discussion}

The main arenas where pesticides are known to be used most commonly are industries, health care, and agriculture to eliminate pests. Evidence attests to negative effects of insecticides and pesticides on male and female fertility, while little is known about other health problems of these compounds, particularly on the reproductive function of women (Sharma et al., 2015). Reportes have been indicative of morphological and molecular changes in the ovaries due to pesticide exposure and poisoning, which could induce various reproductive anomalies, such as cycle irregularities, early puberty, and early senescence. Furthermore, direct exposure is speculated to cause infertility in women and ovarian insufficiency (Armenti et al., 2008).

This is the first study addressing the effect of ALA in GLP-treated GCs on the human folliculogenesis genes. This study showed that GLP pesticide induces toxic effects and causes necrosis, lysis, and GCs disintegration on the female reproductive system. Results showed concentrations of ALA and synergistic group reduced these characteristics and morphological changes in GCs. These features and morphological changes in the cells probably indicate the apoptosis of GCs. Also, our finding showed that exposure to GLP concentrations induced apoptosis on GCs, which might lead to dysfunction of the reproductive system. Further, treatment with ALA concentration reduced morphological change, probably apoptosis, and increased granulosa cell growth.

GLP is the most common herbicide used in the world. As GLP-based herbicides (GBHs) are used in large scale, scientists are concerned with their toxic effects on animals and plants (Benbrook 2016; Duke \& Powles 2008). GLP inhibits 5-enolpyruvyshikimate-3-phosphate synthase (EPSPS), which produces the amino acids tyrosine, tryptophan, and phenylalanine, thereby disrupting the shikimic acid pathway which is found only in plants and various microorganisms (Benbrook 2016). Recent studies showed that GLP is associated with many diseases such as cancer, metabolic disorders, endocrine disruption, imbalanced 
intestinal flora, autism spectrum disorders, and infertility; in addition, it mediates DNA damages, alters gene expression, and causes mutations (WHO 2017; Zhang et al., 2019).

According to Mokhtar et al. (2013), hormonal dysregulation in rats is an adverse consequence of pesticide exposure, which leads to the altered state of progesterone and estrogen. Among the other complications in the mentioned study were endocrine disruption, higher follicular atresia, and lower ovulation (Mokhtar et al., 2013). Moreover, exposure of male rats to pesticides led to increase in abnormal sperm cell count, caused epididymal hypospermia, testicular degeneration, and reduced the weight of testes (Sverdrup et al., 2012). The previous study showed that ovarian follicles, corpus luteumas as well as the number of atretic follicles were reduced in rats exposed to insecticides made of carbamate. Furthermore, evidence suggests that thiodicarb exposure may cause a significant weight loss in reproductive organs of female mice. Other complications associated with thiodicarb exposure have been reported to be ovarian follicular degeneration, while higher follicular atresia as a result of exposure to this pesticide has also been related to inhibition of RNA synthesis of the follicle cells (Matter et al., 2000). According to the literature, the reproductive alterations and distress caused by malathion exposure in the frequency of antral follicle GC apoptosis in the ovaries is positively associated with the granulosa cell apoptosis and lipid peroxidation following malathion exposure (Bhardwaj et al., 2014).

ALA is a natural chemical and an anti-inflammatory and an antioxidant solid agent which is synthesized from octanoic acid and cysteine in human and animal mitochondria. Studies showed that radical effects of ROS are eliminated as ALA fights oxidative stress (Armagan et al., 2015). Therapeutically, ALA is effective on many diseases associated with oxidative stress, including ischemia-perfusion injury, diabetes, cataract formation, neurodegeneration, HIV activation, and radiation damage (Huang et al., 2007; Karapinar et al., 2017). ALA is associated with several diseases, including infertility (Sumathi et al., 2004). Although several studies addressed the effect of ALA on follicle maturation, little is known about the effect of ALA on expression of follicles regulating genes and apoptotic-induced genes; thus, further studies are required. Past studies showed that supplementation with ALA in ovum (IVM) and embryo (IVC) media increased egg nuclear maturation and embryonic growth in pigs. Therefore, their findings show that ALA may improve embryonic system of pigs in vitro and increase pig reproductive performance in the diet (lee et al., 2017).

During development, some genes are activated which are unique to certain deformation during follicular development. These genes are expressed in atretic follicles in connection with reduced proliferation which is a characteristic of this stage. It has been suggested that these genes can be used as biomarkers to detect follicular atresia (Nivet et al., 2009; Bunel et al., 2014). This study demonstrates the effect of GLP and ALA pesticides on expression of folliculogenic genes in human GCs. Also, analysis of gene expression using Real-Time PCR showed that Vnn1 and STAR expressions decreased significantly compared to the control group in the GLP group. In contrast, FOXO1 and NOX4 expressions increased compared to the control group in the GLP group. 
Some genes contribute to development of the reproductive system and are necessary gonads, including the testes and ovaries one of the genes involved in the vnn1 gene. Vanin-1 (Vnn1) belongs to a protein family that has a minimum of three members in humans, including Vanin-1, Vanin-2, and Vanin-3 (Martin et al., 2011). Previous studies have indicated that Vnn1 gene expression leads to a higher follicle diameter and function of time given that such genes have been recognized as the possible indices of follicle differentiation and growth and are also essential to maximum competence (Nivet et al., 2013). At the same time, regulation of the tissue response to oxidative stress is attained by Vnn1 (vanin1) through the modulation of the GSH reservoirs in mice (Berruyer et al., 2015). In this regard, Girard et al. identified the oxidative stress response of $\mathrm{Vnn} 1$, which in turn led to the lower expression of gene in proliferation of cell; the subsequent disruption was related to oxidative stress and GC atresia (Girard et al., 2015). Previous research has also denoted that male germ cell development requires proper conditions, which are provided by the expression of Vnn1 (Vanin-1), so that the gonads could be developed in mice (Megan et al., 2005). This study showed that Vnn1 expression levels decreased with GLP concentrations, indicating atresia as a sign of follicle integrity.

As mentioned earlier, FoxO is a subgroup of transcription factors, which considerably contributes to regulation of a wide-range of cell functions, such as apoptosis, DNA repair, cell proliferation, cell cycle arrest, longevity, stress response, metabolism, senescence, and cell differentiation (Barthel et al 2005). The role of FoxO has been highlighted in mammalian ovaries. On the same note, critical mediators is another terms used to refer to FoxO transcription factors, and among other key functions of these factors are cell apoptosis and oxidative stress regulation. In this regard, the results obtained by Matsuda et al. demonstrated the increased levels of FOXO1 in the GCs of developing follicles (Matsuda et al., 2011). Shen's observations showed that apoptotic signals increased in correlation with higher Fox 01 expression in Mouse granulosa cells (MGCs) of oxidant-treated mice (Shen et al., 2012). Moreover, MGC apoptosis was reported to improve in the rats owing to the activity of the Fox01 mutant and Fox01 (wild-type) overexpression, while the decreased expression of Fox01 prevented apoptosis of the MGCs induced by the oxidant (Shen et al., 2012). Studies on various mammal cells showed that Fox01 activates apoptosisrelated gene expression and induces apoptosis (Burgering et al., 2003). As real-time PCR analysis used in this study showed, FoxO1 expression did not increase significantly in GCs which were exposed to GLP pesticide. Simultaneously, the expression of this gene has decreased in the groups treated with ALA and synergistic. Fox01, expressed in mice ovarian follicles, particularly GCs, induces apoptosis in GCs, indicating that it identifies follicular atresia. Real-time PCR analysis of this study also showed that Fox 1 expression was insignificantly increased in GCs exposed to GLP, which is consistent with the results of shen et al.

NOX4, which is a gene from NOX family, is involved in many cellular systems and ROS. NOX2 and NOX4 isoforms have been shown to produce ROS in the ovary (Chen et al., 2014). NOX 4 and NOX 5 are isoforms expressed in human GCs (Kampfer et al., 2014). Studies show that NOX4 existing in GCs leads to production of ROS, which may be related to female fertility factors. Egg maturation is regulated by NOX-derived ROS which is the second messenger molecule in protein kinase $\mathrm{C}$ signaling pathway. Also, our results showed that NOX4 expression insignificantly increased in GCs exposed to GLP pesticide. In 
contrast, the expression of this gene has decreased in the groups treated with ALA and synergistic. The study by Tousson in 2018 showed that treatment with different concentrations of grape extract reduced ROS content and NOX4 mRNA expression, while opposite was seen in human GCs. Because the main NOX enzyme expressed in GCs is NOX4, ROS content can be regulated by antioxidants in human GCs, possibly through transcriptional effect of NOX4 (Tousson et al., 2018). ROS cell content and NOX4 mRNA expression are reduced by antioxidants, while leaving no effect on cell proliferation and survival. In contrast, environmental stimuli disrupt cell proliferation and stop G1 cell cycle. These data are consistent with decreased cyclin D2 protein expression and increased CDKI and p21 and 27 mRNA expression (Maraldi et al., 2016). In addition, in the latter case, external stimuli cause cell death, increased cleftcaspase-3 and BAD phosphorylation levels, and severe inhibition of Akt phosphorylation levels.

STAR (Steroidogenic Acute Regulatory Protein) is one of the steroidogenic enzymes (such as Cyp11a1, STAR, Cyp19a1, and 3ß-HSD) regulated and expressed in GCs. The most important steroid enzyme in GCs is the STAR gene, which is involved in the transfer of cholesterol from the outer mitochondrial membrane to the inner membrane and its conversion to pregnenolone. Pregnenolone produces progesterone. Alterations in steroid enzyme expression and hormone secretion in GCs are the main cause of impaired follicular development and maturation, leading to premature or premature cessation of follicle growth in poly syndrome ovarian cysts (PCO) (Bakhshalizadeh et al., 2017). Ovarian steroid regulatory genes keep the follicle steroid hormones stable and contribute to follicle growth and maintain follicle and luteal function (Guo et al.,2019). The CYP11A1 and STAR genes are the most important regulatory genes known to be involved in the expression of progesterone production and metabolism in the ovaries (LeeThacker et al., 2010). The present study showed that STAR expression decreased in exposure to GLP concentrations, while this gene expression has reduced in the groups treated with ALA and synergistic. These negative effects were also connected with higher levels of oxidative stress and morphological changes; they suggest that external stimuli could exert some detrimental effects on ovarian cells. Also, antioxidants improved steroidogenesis by increasing expression. CREB, STAR protein, and MAPK ERK1 / 2 phosphorylation are related. Previous studies have shown that antioxidants in human GCs lead to a significant increase in secretion of progesterone and estradiol as well as STAR protein which contributes to transport of cholesterol from the outer mitochondrial membrane (Chen et al., 2014).

In general, antioxidants are powerful tools to reduce the severe effects of oxidative stress on cell systems. ALA is one of the antioxidants that can directly scavenge free radicals and maintain the innate cellular antioxidant defense system (Hatami et al., 2014).previous studies showed that ALA improves testicular function in mice exposed to chemical compounds through its steroidogenic and antioxidant properties (Zoheir et al., 2016).The ability of ALA to scavenge free radicals directly or indirectly by retention of antioxidants, including GSH recycling, may act as a shield and protect tissues against oxidative damage caused by arsenic. It is suggested that caspase- 3 activation acts as one of the downstream oxidative stress events and is involved in apoptosis. Thus, a decrease in caspase- 3 mRNA in arsenic-treated mice's testes may reflect the anti-apoptotic potential of ALA (Zoheir et al., 2016). A significant improvement in steroidogenesis, spermatogenesis, and improvement in sperm maturation events in ALA-treated mice may indicate a beneficial effect on Sertoli and Leydig and epididymal cells against arsenic-induced 
oxidative stress. Also, show study, ALA may reduce apoptosis of secondary preantral follicles; the effect of ALA on expression of genes which regulate follicle development (such as FSHR, LHCGR, IGF-1, BMPR1a, TGF $\beta R 1, T G F \beta 1$, ActRIIB, GDF9 and Activin A) and apoptotic-induced genes (BCL2-associated X protein (BAX) and C-Myc) was evaluated using secondary preantral follicles of bovine. Other studies also showed that the growth rate of secondary preantral follicles is improved by adding ALA to culture medium. Once the culture was over, ALA increased the numbers of living follicles. The reason is that follicular growth-regulating genes are upregulated and apoptosis-induced genes are down-regulated (partiman et al., 2017). The present study shows that ALA and the synergistic concentration group have no destructive effect on the female reproductive system and have antioxidant properties.

\section{Conclusion}

Results show that exposure to GLP causes morphological changes in the structure of GCs, which may interfer with reproductive system fonction. At the same time, treatment with ALA antioxidant reduces the destructive effects of GLP pesticide on GCs. This study also showed that GLP reduced Vnn1, STAR expressiona and increased FoxO1, NOX4 expressions, while treatment with ALA antioxidant increased expression of Vnn1, STAR, and reduced Fox01, NOX4 genes. This study showed that ALA and the synergistic group concentrations could be reliable antioxidants due to minimal damage to the female's reproductive system.

\section{Declarations}

Ethical Approval: This research was approve by the Ethics Committee of Birjand University of Sciences (IR.BUMS.REC.1398.393) and was carried out in accordance with the latest version of the Declaration of Helsinki.

Consent to Participate: Not Applicable

Consent to Publish: Not Applicable

Conflicts of interest: None declared.

Authors' Contributions: Study design and conception were accomplished by all the authors. S.K.K., A.A., F.GH., and E.M.M; performed material preparation, samples collection, and data analysis. S.K.K. prepared the initial draft of the manuscript. Necessary feedback was provided by the authors in the previous version of the manuscript, and the final manuscript was read and approved by all the research team members. E.M.M. and A.A.; Supervised the research.

Funding: This work was given a grant (grant Number: 393) from Birjand University of Medical Science (Miri-Moghaddam Ebrahim), Iran. 
Availability of data and materials: All data generated or analysed during this study are included in this published article [and its supplementary information files].

Acknowledgments: We gratefully appreciate the contributions of data collection team and people who participated in this study. The authors specially thank the Research Deputy of Birjand University of Medical Sciences (BUMS), Birjand, Iran, Milad Infertility Center, Mashhad University of Medical Sciences, Mashhad, Iran and Animal Development and Applied Biology Research Center of Mashhad Branch, Islamic Azad University, Mashhad, Iran for their support of this work.

\section{References}

Armagan I, Bayram D, Candan IA, et al (2015) Effects of pentoxifylline and alpha lipoic acid on methotrexate-induced damage in liver and kidney of rats. Environ Toxicol 39:1122-1131

Armenti AME, Mahakali ZA, Passantino L, Uzumcu M (2008) Developmental methoxychlor exposure affects multiple reproductive parameters and ovarian: folliculogenesis and gene expression in adult rats. Toxicol Appl Pharmacol 233:286-296

Bakhshalizadeh S, Amidi F, Alleyassin A, Soleimani M, Shirazi R, Shabani Nashtaei M (2017) Modulation of steroidogenesis by vitamin D3 in granulosa cells of the mouse model of polycystic ovarian syndrome. 63(3): 150-161

Barthel A, Schmoll D, Unterman TG (2005) FoxO proteins in insulin action and metabolism. Trends Endocrinol Metab 16:183-189

Benbrook CM (2016) Trends in glyphosate herbicide use in the United States and globally. Environ Sci Eur $28: 3$

Berruyer C, Martin FM, Castellano R, Macone A, Malergue F,Garrido-Urbani S, et al (2004)Vanin-1K/K mice exhibit a glutathione-mediated tissue resistanceto oxidative stress. Mol Cell Biol 24: 7214-7224

Bhardwaj JK, Saraf P (2014) Malathion-Induced Granulosa Cell Apoptosis In CaprineAntral Follicles: AnUltrastructural And Flow Cytometric Analysis. J Microscopy and Microanalysis 20: 1861-1868

Burgering BM, Medema RH (2003) Decisions on life and death: FOXO forkhead transcription factors are in command when PKB/Akt is off duty. J Leukoc Biol 73:689-701

Bunel A, Nivet A, Blondin P, Vigneault C, Richard F, Sirard M (2014) Cumulus cell gene expression associated with pre-ovulatory acquisition of developmental competence in bovine oocytes. Reprod Fertil Dev 26:855-865

Chen Q, Zhang W, Ran H, Feng L, Yan H, Mu X, Han Y, Liu W, Xia G, Wang C (2014) PKCס and $\theta$ possibly mediate FSH-induced mouse oocyte maturation via NOXROS-TACE cascade signaling pathway. 9(10):1-8 
Chevrier C, Warembourg C, Gaudreau E, Monfort C, Le Blanc A, Guldner L, Cordier S (2013) Organochlorine pesticides, poly-chlorinated biphenyls, seafood consumption, and time-to-pregnancy. Epidemiology 24:251-260

Chiu $Y$ et al (2015) Fruit and vegetable intake and their pesticide residues in relation to semen quality among men from a fertility clinic. Hum Reprod 30:1342-1351

Den Hond E et al (2015) Human exposure to endocrine disrupting chemicals and fertility: a case-control study in male subfertility patients. Environ Int 84:154-160

Duke S.O, Powles S.B. (2008) Glyphosate: a once-in-a-century herbicide. Pest Manag. Sci. 64: $319-325$

García J, Ventura MI, Requena M, Hernández AF, Parrón T, Alarcón R (2017) Association of reproductive disorders and male congenital anomalies with environmental exposure to endocrine active pesticides. Reprod Toxicol 71:95-100

Girard A, Dufort I, Douville G, Sirard MA (2015) Global gene expression in granulosa cells of growing, plateau and atretic dominant follicles in cattle. J Reprod Biol Endocrinol 13:1-15

Guo YX, Zhang GM, Yao XL, Tong R, Cheng CY, Zhang TT, Wang ST, Yang H, Wang F (2019) Effects of nitric oxide on steroidogenesis and apoptosis in goat luteinized granulosa cells. 126:55-62

Gupta RK, Schuh RA, Fiskum G, Flaws JA (2006) Methoxychlor causes mitochondrial dysfunction and oxidative damage in the mouse ovary. Toxicology and Applied Pharmacology 216:436-445

Hatami S, Zavareh S, Salehnia M, Lashkarbolouki T, Karimi I (2014) Comparison of oxidative status of mouse pre-antral follicles derived from vitrified whole ovarian tissue and vitrified preantral follicles in the presence of alpha lipoic acid. J Obstet Gynaecol Res 40(6):1680-8

Huang H, Tindall DJ. (2007) Dynamic FoxO transcription factors. J Cell Sci 120: 2479-2487

Kampfer C, Saller S, Windschüttl S, Berg D, Berg U, Mayerhofer A (2014) Pigment-Epithelium Derived Factor (PEDF) and the human ovary: a role in the generation of ROS in granulosa cells 97: 129-136

Lee JE, Hwangbo Y, Kim HY, Lee WH, Cheong HT, Yang B , Park CK (2017) Effect of Alpha-Linolenic Acid on Oocyte Maturation and Embryo Development in Pigs 21: 2005-2013

Lee-Thacker S, Choi Y, Taniuchi I, Takarada T, Yoneda Y, Ko C, Jo M (2018) Core Binding Factor $\beta$ Expression in Ovarian Granulosa Cells Is Essential for Female Fertility 159(5):2094-2109

Maraldia T, Rescab E, Nicolic A, Berettia F, Zavattia M, Capodannoc F, Morinic D, Palombac S, Salac G.B, Polanto A.D (2016) NADPH oxidase-4 and MATER expressions in granulosa cells: Relationships with ovarian aging. Life Sciences 162: 108-114 
Matsuda F, Inoue N, Maeda A, Cheng Y, Sai T, Gonda H, et al (2011) Expression and function of apoptosis initiator FOXO3 in granulosa cells during follicular atresia in pig ovaries. J Reprod Dev 57:151-158

Matter FE, Farid NM, Rageb AF (2000) A histochemical study on the ovaries of rat treated with an insecticide. "Carbaryl"Egypt J Histol 4:87-92

Megan JW, Jeyasuria P, Keith LP, Koopman P (2005)The transcription factors steroidogenic factor-1 and SOX9 regulate expression of Vanin-1 during mouse testis development. J Biol chem 18: 5917-5923

Mokhtar HI, Abdel HA, Elmazoudy RH, Abdelwahab WM, Saad MI ( 2013) Effect of methomyl on fertility, embryotoxicity and physiological parameters in female rats. J App Pharm Sci 3 (12): 109-119

Mostafalou S, Abdollahi M (2017) Pesticides: an update of human exposure and toxicity. Arch Toxicol 91:549-599

Milesi MM, Lorenz V, Pacini G, Repetti MR, Demonte LD, Varayoud J, Luque EH(2018) Perinatal exposure to a glyphosate-based herbicide impairs female reproductive outcomes and induces secondgeneration adverse effects in Wistar rats. Arch Toxicol 92 (8): 2629-2643

Navari-Izzo F, Quartacci MF, Sgherri C (2002) Lipoic acid: a unique antioxidant in the detoxification of activated oxygen species. Plant Physiol Biochem 40:463-470

Nivet AL, Vigneault C, Blondin P, Sirard MA (2013) Changes in granulosa cells gene expression associated with increased oocyte competence in bovine. J Reproduction 145:555-565

Organization WH. WHO (2010) laboratory manual for the examination and processing of human semen.

Prathima P, Pavani R, Sukeerthi S, Sainath SB (2017) $\boldsymbol{\alpha}$ - Lipoicacid inhibits testicular and epididymal oxidative damage and improves fertility effica cyinarsenic-intoxicatedrats. JBiochemMolToxicol e22016

Rappazzo KM, Warren JL, Meyer RE, Herring AH, Sanders AP, Brownstein NC, Luben TJ (2016) Maternal residential exposure to agricultural pesticides and birth defects in a 2003 to 2005 North Carolina birth cohort. Birth Defects Res Part A Clin Mol Teratol 106:240-249

Saleh RA, Agarwal A (2002) Oxidative stress and male infertility: from research bench to clinical practice. J Androl 23:737-752

Sargazi Z, Nikravesh MR, Jalali M, Sadeghnia H, Rahimi FA, Mohammadzadeh L (2015) Gender-Related Differences in Sensitivity to Diazinon in Gonads of Adult Rats and the Protective Effect of Vitamin E. International Journal of Women's Health and Reproduction Sciences 3:40-47

Sharma D, Sangha GK, Khera KS (2015) Triazophos -induced oxidative stress and histomorphological changes in ovary of female Wistar rats. J pesticide Biochemistry and physiology 117:9-18 
Shen M, Lin F, Zhang J, Tang Y, Chen WK, Liu H (2012) Involvement of the up-regulated Fox01 expression in follicular granulosa cell apoptosis induced by oxidative stress. J Biol chem 31: 25727-25740

Soylu Karapinar O, Pinar N, Özcan O, Özgür T, Dolapçığlu K (2017) Protective effect of alpha-lipoic acid in methotrexate-induced ovarian oxidative injury and decreased ovarian reserve in rats. Gynecol Endocrinol 33(8):653-659

Sverdrup LE, Bjorgr C, Eklo OM, Grung M, Kallqvist T, Klingen I, Lag M, Ropstad E, Ovrebo S (2012) Risk assessment of the insecticide Movento 100 SC with the active substance spirotetramat.13-203

Sumathi R, Baskaran G, Varalakshmi P (1996) Relationship between glutathione and DL alphalipoic acid against cadmium-induced hepatotoxicity. Jpn J Med Sci Biol 49(2): 39-48

Tousson E, Elgharabawy R.M, Elmasry T.A (2018) Grape Seed Proanthocyanidin Ameliorates Cardiac Toxicity Induced by Boldenone Undecylenate through Inhibition of NADPH Oxidase and Reduction in the Expression of NOX2 and NOX4. Oxidative Med. Cell. Longev 10(1155): 34-38

WHO (2017) Sexual and reproductive health: infertility is a global public health issue. http://www.who.int/reproductivehealth/ topics/infertility/perspective/en/ Accessed 22 Dec 2017

Zhang JW, Xu DQ, Feng XZ (2019) The Toxic Effects and Possible Mechanisms of Glyphosate on Mouse Oocytes. Chemosphere 237:1-10

Zoheir K.M.A, Gamaleldin I. H, Ahmed A.A, Yang L, Xiang L, Aixin L, Ahmed A. A, Abdel H.H (2016) Effect of alpha lipoic acid on in vitro development of bovine secondary preantral follicles. Theriogenology 16:30426-5

Ziv-Gal A, Flaws JA (2016) Evidence for bisphenol A-induced female infertility: a review (2007-2016). Fertil Steril 106:827-856

\section{Tables}

Table 1 The sequence of primers used for genes expression detection by Real-time PCR. 


\begin{tabular}{|c|c|c|c|}
\hline Gene & $\begin{array}{lll}\text { Forward } \quad 5 \square \rightarrow & 3 \square\end{array}$ & Reverse $\quad 5 \square \rightarrow 3 \square$ & Annealing $\left({ }^{\circ} \mathrm{C}\right)$ \\
\hline FOXO1 & TCACCCAGCCCAAACTACCAA & CTTCAAGAGTCCAGGCGCACA & 58 \\
\hline Vnn1 & CTATGCCAGCAGTATAGAAGC & CGCAAGTGTTTAAATTAGTCG & 58 \\
\hline NOX4 & CAGTCACCATCATTTCGGTCA & GATGAACCCCAAATGTTGCTT & 58 \\
\hline STAR & CCACAGACTTCGGGAACATGCC & ¿CCACCCCTTGAGGTCGATGC & 58 \\
\hline GAPDH & AAGCTCATTTCCTGGTATGACAACG & TCTTCCTCTTGTGCTCTTGCTGG & 59 \\
\hline
\end{tabular}

\section{Figures}
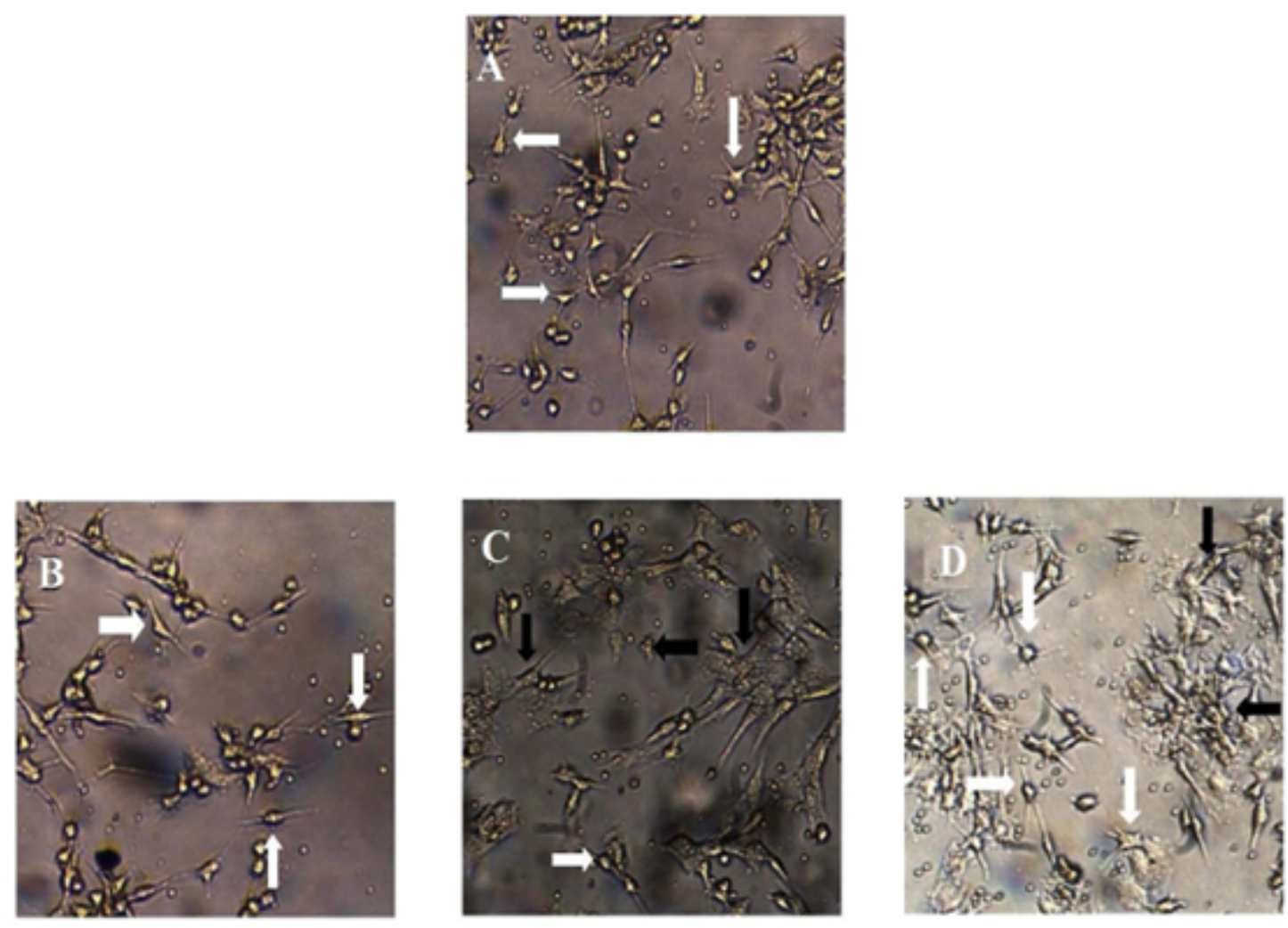

\section{Figure 1}

Cytomorphological effects of glyphosate, alpha-lipoic acid, and synergetic combinations or GLY+ ALA group on GCs, A: control group B: glyphosate $250 \mu \mathrm{g} / \mathrm{ml}$, C: alpha-lipoic acid $50 \mu \mathrm{g} / \mathrm{ml}, \mathrm{D}$ : and 
combinations glyphosate + alpha-lipoic acid group (250 glyphosates +50 alpha-lipoic acid), the morphology of GCs $24 \mathrm{hr}$ after treatment (20X magnification).

FOXO
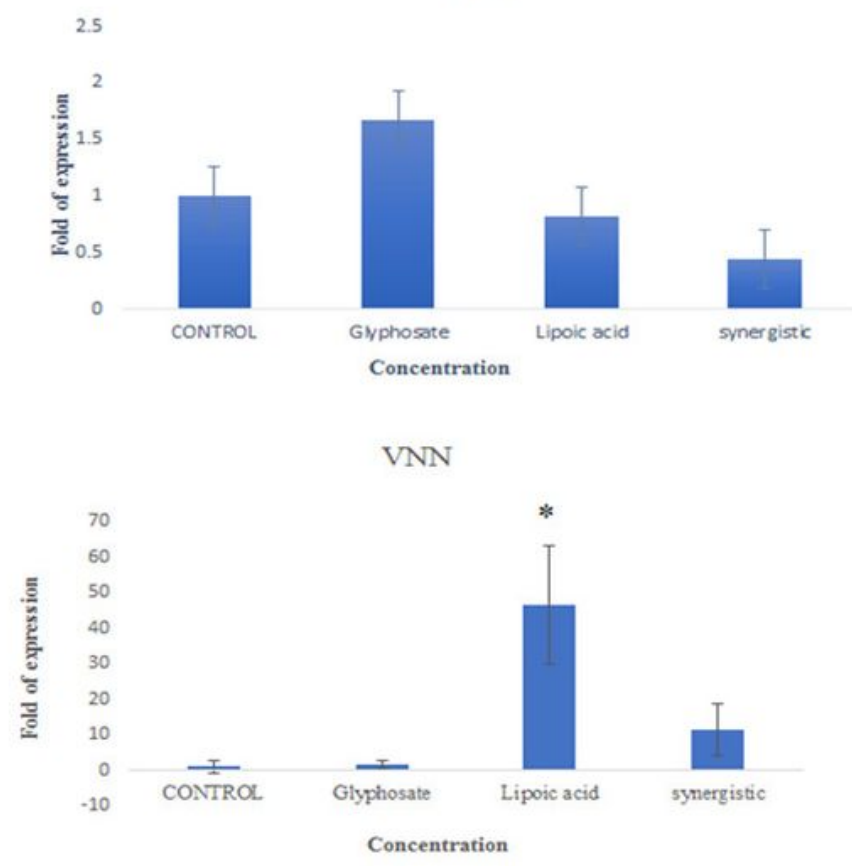

NOX-4
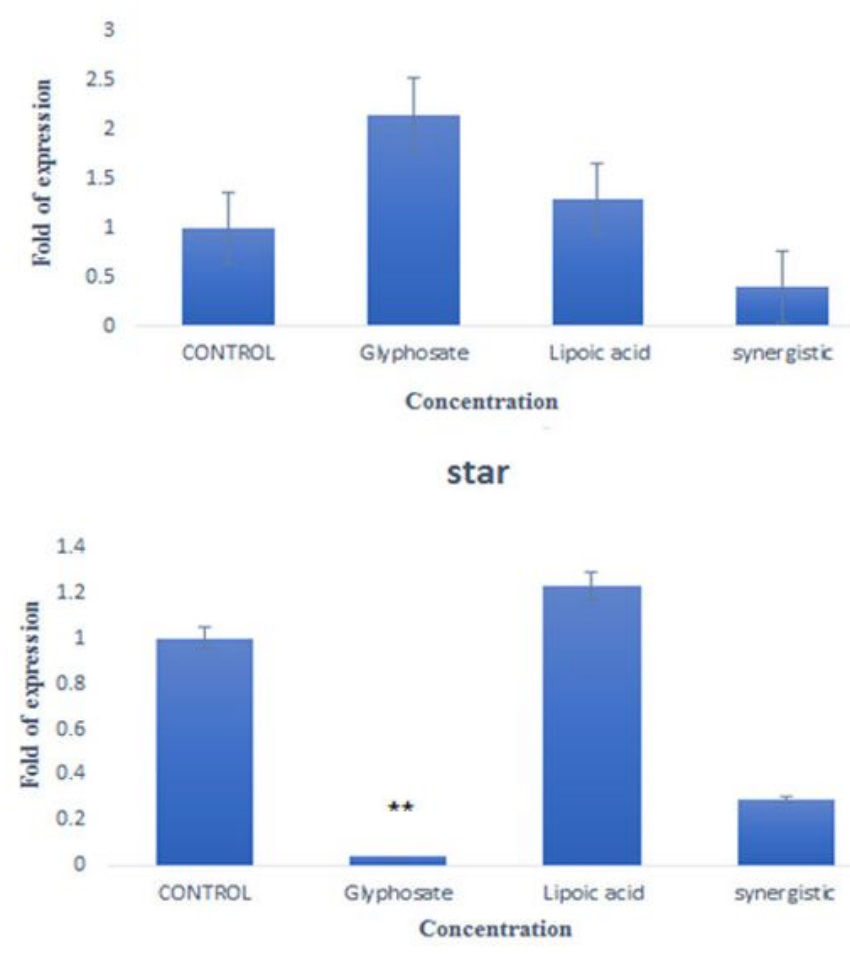

Figure 2

Real-time PCR analysis of representative genes, Fox01, NOX4, Vnn1, and STAR genes expression in GCs treated with GLP concentrations, alpha-lipoic acid, and synergetic combinations or glyphosate + alpha- 
lipoic acid group on GCs. Data are expressed as mean \pm SD. The values are significantly different (*Pvalue $<0.05)(\star \star P-v a l u e<0.01)$ in comparison with the controls. 